\title{
SOME IDEAS ON DIVERSITY, INCLUSION AND METAPHORIZATION IN POLYGLOT STUDIES
}

\author{
Nastasia Britsyna \\ Moscow State Institute of International Relations (University) \\ 76, Prospekt Vernadskogo, Moscow, 119454, Russia
}

$\mathrm{D}$ ear colleagues and guests, it is a great pleasure to be invited to this seminar, especially in the capacity of a MGIMO faculty member. It seems that the venue could not be more apt, given the multilingual environment of our university, which has been honoured for many decades.

To begin with, the global-minded and digitally driven world with its ever-expanding human migration is bringing high expectations for academia in search for helpful solutions and, hence, newfound emphases in sciences. Polyglottery, in particular, is given fresh impetus, which logically stems from where the international community is heading. Never have the concepts of diversity and inclusion been as relevant as they are today: abundant cultural backgrounds are being united in the framework of international organizations, social movements and businesses. Essentially, these concepts are emerging in the fundamental papers of global organizations (such as manifold UN agendas) and corporate mission statements of large and small companies. This invigorates interest in multilingualism as a field which truly embodies the two said concepts being "inclusive" and "diverse" by nature. This interest cannot be explained only by the practical value of mastering different languages for business purposes, though it is definitely a powerful factor. Another significant force at work is a complex cultural fabric that weaves together countries across the globe (a consequence of globalization), and it is multilingualism that enables a richer cultural experience and deeper understanding of cultures given the experiential and cognitive nature of every language.

The seminar provides a platform to discuss the main subject areas for fruitful research in polyglottery. For my part, I would like to emphasize metaphors in their cognitive-linguistic dimension. With a powerful burst of scientific interest in cognitive sciences, polyglottery now has the opportunity to greatly benefit from the field. For instance, it could enrich modern studies with more data on how human brain operates information and arranges it in its "conceptualizing systems", "maps of reality", "conceptual worldviews", "mental spaces", etc., depending on the theory we choose. I must highlight that the language of science itself is very metaphorical: prime examples can be found even in the most unambiguous terms such as those of natural sciences. For instance, biologists and medical doctors systematically use metaphors when they refer to "the nasal bridge", "a macrophage stretching its arms", "anatomy trains" and "astrocytes".

In this regard, I would like to reiterate the point that has already been made by Professor Minoru Ohtsuki: in many cases, there is no other way to express these ideas except for a metaphorical one. This leads us to the conclusion that metaphors become something more than just tools that make our perception of complex and abstract objects/phenomena easier. We see that in some cases they are the only option we can think of. Thus, polyglottery is an intriguing window on linguistic patterns that might shine 
a light on how humans establish connections between referents which in reality are not connected at all. Various methods of metaphorical analysis in multiple languages, of different families, could elucidate or at least bring us closer to the complex issues of collecting and storing linguistic data. In a sense, analyzing metaphors is a new sort of linguistic data "mining" that enables one to extract primary mechanisms by which our multifaceted world is represented. This, in its turn, can facilitate NLP studies (by this I mean natural language processing, with no reference to neuro-linguistic programming) and related domains of human knowledge.

Another point that should be given thoughtful consideration is student engagement. Building young scholar networks is definitely conducive to the advance of science as it promises brand new perspectives and empowers young talents. The latter deserves special attention: any "unusual" contact with the language (not being part of ordinary class activities) fosters student awareness and interest. As scientific tasks are characterized by a heuristic element, they are way out of the "usual" and monotonous. Discoveries and conclusions that young scholars could share with each other may create a more positive state of mind by eliminating perceived barriers in language acquisition. Independent thinking and dealing with languages could boost the learner's confidence as research gives them more sense of control over the language tools they can unpick, break down and then assemble in a unique way to produce successful communication. This, in turn, might result in better performance in the classroom and beyond. That is why I am so happy to see Professor Michio Tajima's future graduate student who decided to join this meeting, potentially signaling the start of greater youth involvement.

In that spirit, the seminar seems to be a fertile ground for cooperation among those who show deep commitment to language science, language learning and language acquisition. Polyglot studies can truly equip us with more effective communication and education strategies as well as allow us to look through the kaleidoscope of cultures and cognitive patterns and thus to better understand ourselves.

Nastasia Britsyna, MA in Linguistics, is Lecturer at Department of English No.3, Moscow State Institute of International Relations (MGIMO). Research interests: cognitive linguistics, conceptual metaphors, discourse analysis, media discourse, academic discourse, semantic analysis, pragmatics. E-mail: nast.britsyna@mail.ru 